\title{
A propósito de "Terapia eléctrica de las arritmias cardiacas"
}

\begin{abstract}
Al igual que las distintas ramas de la ciencia, la Medicina, esa amalgama de arte y ciencia, fue descubriendo, lentamente a través de muchos siglos, las bases elementales de cuerpo humano: su estructura y función.

Pero durante los últimos 100 ó 50 años como una vertiginosa cascada de dominó, un hecho condujo a otro y al otro; no solo nos conoceríamos en lo macro sino en lo microscópico, luego en lo genético $\mathrm{y}$ ahora avanzamos en lo molecular.
\end{abstract}

Al comprender mejor nuestras enfermedades, se crean nuevos métodos diagnósticos, cada vez más complejos, cada vez más exactos y paralelamente se desarrollan nuevos tratamientos, algunos quedan, otros se descartan, pero lo cierto es que ningún ser humano puede dominar el conocimiento médico actual. La Medicina se fragmenta entonces es un sinnúmero de especialidades y éstas es subespecialidades. Ya casi no se es solo internista, sino neumólogo y éste puede ser pediatra o broncoscopista intervencionista, ecocardiografista $o$ electrofisiólogo.

El trabajo diario, la falta de incentivos, los altos costos y otras razones continuamente nos dificultan mantenernos al día, aún en nuestra propia área. Solo la asequibilidad de las publicaciones escritas y cibernéticas puede ayudarnos a ello.

Acta Médica ofrece en este número un artículo sucinto y claro de aparatos y métodos disponibles en el mercado para el tratamiento no medicamentoso de las arritmias cardiacas. La Dra. Esquivel y el Dr. Gutiérrez nos presentan aquí un desfile de aparatos electrónicos cardiacos con funciones y características tan inteligentes que logran dominar el corazón y hacer que este obedezca rompiendo arritmias o contrayéndose "secuencialmente", es decir fisiológicamente, primero los atrios luego los ventrículos. También nos describen los autores otros métodos invasivos utilizados en la destrucción de vías o tractos accesorios causantes de arritmias fatales tratados antes mediante procedimientos mas cuentos y mórbidos.

Aunque durante los años 1980 y 1907 se describió el sistema de conducción del corazón, cuando His, Keith y Flack y Tawara aportaron cada uno de sus descubrimientos individuales acerca de los elementos anatómicos y del funcionamiento de los mismos en conjunto (1), los tratamientos aplicables a los trastornos del ritmos cardiaco que causaron un impacto profundo en los resultados, no se descubrieron sino hasta unos 50 años más tarde. Durante esos primeros años y con instrumentos arcaicos, algunos científicos estimulaban en el laboratorio ancas de rana y corazones aislados.

Pero había que esperar la comunicación de unos clínicos con otros, entender los hallazgos anatomopatológico, diseñar instrumentos de tiempo confiables con los cuales medir el pulso y desarrollar la electrocardiografía dentro de otras cosas, para entender y separar las arritmias.

Los nombres de Morgagni, Stonkes, Adams, Krimer, Hyman forman parte de esa primera ola de científicos, luego vinieron Dammann, Zoll, Lillehei y Furman dentro de otros, que aplicaron estos electrodos y aparatos externa e internamente a los pacientes con trastornos del ritmo ${ }^{2}$.
En nuestro país, como en todo el mundo, los marcapasos fueron primero colocados por cirujanos, el grupo del Dr. Longino Soto Pacheco, en el Hospital México, que practicaba ya la cirugía cardiaca bajo circulación extracorpórea como un procedimiento de rutina, y el que al principio del programa operaba niños y adultos, pronto adquirió mucha experiencia en esta tecnología ${ }^{3,4}$ y hoy décadas después mantiene miles de pacientes en control.

Como bien describen los autores en su artículo, los marcapasos hoy son solo parte de esa de gama de implementos, todos ellos son insertados corrientemente por los cardiólogos electrofisiólogos. La electrofisiología es una subespecialidad verdaderamente compleja que requiere no solo de equipos sofisticados y caros sino de profesionales profundamente conocedores de la fisiología y electrocardiografía y también hábiles en la manipulación de catéteres y electrodos y en la interpretación de los trazos externos e intracavitarios ${ }^{5,6}$. Ocasionalmente se debe de acudir a la ayuda de los cirujanos si hay problemas con la fijación interna de los electrodos u otras complicaciones.

En Costa Rica existe ya un grupo de profesionales especializados que han impulsado esta rama de la medicina ${ }^{7,8} \mathrm{y}$ del cual esperamos seguir aprendiendo.

\section{Referencias}

1. Word P. Diseases of Herat and circulation. Chap. VI. Disorders of the cardiac rhythm. JB Lippincot Co. Philadelphia. 1983, p:1306.

2. Tyers FO. Cardiac pacemakers and cardiac conduction system abnormalities. En Gibbons Surgery of the Chest. Sabiston y Spencer (edits). Surgery of the Chest. Philadelphia: Sauders. 1983, p:1306.

3. Salazar C. Marcapasos cardiacos. Acta Medic Costarric. 23(3): 267-274

4. Salazar C, Soto L, Brenes C, Castro A, Pucci JJ, Fallas M. Uso de marcapasos cardiacos en niños. Acta Medic Costarric. 1980, 23(3) 235-238

5. Araya V, Electrofisiología intervencionista: procedimientos diagnósticos y terapéuticos en arritmias cardiacas. Rev. Costarr. Cardiol:1999. (1):9-21.

6. Araya V, Gutiérrez O, Cruz F, Electrofisiología intervencionista: experiencias inicial en Costa Rica con procedimientos diagnósticos y terapéuticos en arritmias cardiacas. Rev Costarr Cardiol. 2001, 3(1):22-33.

7. Cruz F,Araya V, Gutiérrez O. Cardiodesfibriladores automáticos implantables. Experiencia inicial en Costa Rica. Rev. Costarric Cardiol. 2001, 3(1):42-48.

8. Cruz F,Araya V, Gutiérrez O, Mainieri J, Arce M. Implante del primer resincronizador cardiaco en Costa Rica. Act. Med.Costarr.2001, 43 Supl.: 17
Dr. Carlos Salazar Vargas
FACS/FACCP/FAHA.
Coordinador Programa de Cirugía Cardiovascular Hospital R.A Calderón Guardia 